\title{
Affine processes are regular
}

\section{Journal Article}

\section{Author(s):}

Keller-Ressel, Martin; Schachermayer, Walter; Teichmann, Josef

Publication date:

2011-12

Permanent link:

https://doi.org/10.3929/ethz-b-000028228

Rights / license:

In Copyright - Non-Commercial Use Permitted

Originally published in:

Probability Theory and Related Fields 151(3-4), https://doi.org/10.1007/s00440-010-0309-4 


\title{
Affine processes are regular
}

\author{
Martin Keller-Ressel • Walter Schachermayer • \\ Josef Teichmann
}

Received: 18 June 2009 / Revised: 22 March 2010 / Published online: 30 June 2010

(C) Springer-Verlag 2010

\begin{abstract}
We show that stochastically continuous, time-homogeneous affine processes on the canonical state space $\mathbb{R}_{>0}^{m} \times \mathbb{R}^{n}$ are always regular. In the paper of Duffie et al. (Ann Appl Probab 13(3):984-1053, 2003) regularity was used as a crucial basic assumption. It was left open whether this regularity condition is automatically satisfied for stochastically continuous affine processes. We now show that the regularity assumption is indeed superfluous, since regularity follows from stochastic continuity and the exponentially affine form of the characteristic function. For the proof we combine classic results on the differentiability of transformation semigroups with the method of the moving frame which has been recently found to be useful in the theory of SPDEs.
\end{abstract}

Keywords Affine processes $\cdot$ Regularity $\cdot$ Characteristic function $\cdot$ Semiflow

Mathematics Subject Classification (2000) $\quad 60 \mathrm{~J} 25 \cdot 39 \mathrm{~B} 32$

M. Keller-Ressel and J. Teichmann gratefully acknowledge the support from the Austrian Science Fund (FWF) under grant Y328 (START prize).

W. Schachermayer and J. Teichmann gratefully acknowledge the support from the Austrian Science Fund (FWF) under grant P19456, from the Vienna Science and Technology Fund (WWTF) under grant MA13 and by the Christian Doppler Research Association (CDG).

M. Keller-Ressel · J. Teichmann ( $\varangle)$

ETH Zürich, D-Math, Rämistrasse 101, 8092 Zürich, Switzerland

e-mail: jteichma@math.ethz.ch

M. Keller-Ressel

e-mail: kemartin@math.ethz.ch

W. Schachermayer

Faculty of Mathematics, University of Vienna,

Nordbergstrasse 15, 1090 Vienna, Austria

e-mail: walter.schachermayer@univie.ac.at 


\section{Introduction}

A Markov process $X$ taking values in $D:=\mathbb{R}_{\geq 0}^{m} \times \mathbb{R}^{n}$ is called affine if there exist complex-valued functions ${ }^{1} \Phi(t, u)$ and $\psi(t, u)$ such that

$$
\mathbb{E}^{x}\left[e^{\left\langle X_{t}, u\right\rangle}\right]=\Phi(t, u) \exp (\langle x, \psi(t, u)\rangle)
$$

for all $x \in D$, and for all $(t, u) \in \mathbb{R}_{\geq 0} \times i \mathbb{R}^{d}$ with $d=m+n$. We also assume that $X$ is stochastically continuous, i.e. $X_{t} \rightarrow X_{s}$ in probability as $t \rightarrow s$.

Stochastic processes of this type have been studied for the first time in the seventies, where (on the state space $D=\mathbb{R}_{\geq 0}$ ) they have been obtained as continuous-time limits of classic Galton-Watson branching processes with and without immigration (see [8]). More recently, affine processes have attracted renewed interest, due to several applications in mathematical finance, where they are used as flexible models for asset prices, interest rates, default intensities and other economic quantities (see [3] for a survey).

The process $X$ is said to be regular, if the functions $\Phi$ and $\psi$ are differentiable with respect to $t$, with derivatives that are continuous in $(t, u)$. This technical condition is of crucial importance for the theory of affine processes, as developed in [3]. Under the assumption of regularity, it is possible to show that every conservative affine process $X$ is a semi-martingale, and in fact to completely characterize all affine processes in terms of necessary and sufficient conditions on their infinitesimal generator. Likewise, regularity allows to represent the characteristic function of $X$ as the solution of so-called generalized Riccati differential equations which determine the fundamental functions $\Phi$ and $\psi$.

Without regularity it is not immediately clear how we can "locally" characterize the process (e.g. in terms of its infinitesimal generator or of its semi-martingale characteristics) and therefore also not clear whether affine processes form a well-parameterized family - a situation which could be compared to the theory of Lévy processes without knowledge of the Lévy-Khintchine formula. For this reason, [3] assume regularity at the very beginning of their classification of affine processes. Without the assumption of stochastic continuity there are simple examples of non-regular Markov processes with the affine property (1.1), based on introducing jumps at fixed (non-random) times; see e.g. [3, Remark 2.11]. In contrast, if one assumes stochastic continuity for an affine process, to the best of our knowledge it was not known in general whether such a process is regular or not. Regularity has been shown in several special cases: [8] show automatic regularity for a single-type continuous branching process with immigration, which corresponds to an affine process with state space $D=\mathbb{R}_{\geq 0}$. [2] show regularity of affine processes under moment conditions. In [10] regularity of an affine process under a mixed homogeneity and positivity condition is shown. The approaches in the two latter publications are all based on the general techniques from [12] for continuous

\footnotetext{
1 We are using upper case $\Phi$ but lower case $\psi$ for consistency with the notation of [3]; see Remark 2.3 for a detailed discussion.
} 
(global) flows of homeomorphisms, which have been extended in [4] to continuous (local) semi-flows.

One of the most fascinating aspects of the regularity problem for affine processes is the close connection to the Hilbert's fifth problem, whose history and mathematical development can be found in [12]. The reason is that the functions $\Phi$ and $\psi$ defined by (1.1) satisfy certain functional equations, namely

$\psi(t+s, u)=\psi(t, \psi(s, u)), \quad \Phi(t+s, u)=\Phi(t, \psi(s, u)) \cdot \Phi(s, u)$, for $s, t \geq 0$

with initial conditions $\psi(0, u)=u$ and $\Phi(0, u)=1$ and for all $u$ in $\mathcal{Q}$, a large enough subset of $\mathbb{C}^{d}$ (see Sect. 2 for the exact definition of $\mathcal{Q}$ ). Such functional equations are relatives of the multiplicative Cauchy functional equation

$$
A(t+s)=A(t) A(s), \quad A(0)=\mathrm{id}_{d},
$$

formulated for instance in a set of $d \times d$ matrices $M_{d}(\mathbb{C})$, simply by defining $\psi(t, u)=$ $A(t) u$. It is well-known that the only continuous solutions of the Cauchy equation are the exponentials $A(t)=\exp (t \beta)$, where $\beta$ is a $d \times d$ matrix. In particular all continuous solutions of (1.3) are automatically differentiable (even analytic) with respect to $t$, with derivatives that are continuous in $u$.

Hilbert's fifth problem asks whether this assertion can be extended to more general functional equations such as (1.2): Assuming that $\psi(t, u)$ and $\Phi(t, u)$ satisfy (1.2), and are differentiable in $u$, are they necessarily differentiable in $t$ ? The problem has been answered positive; for a precise formulation and a proof in the more general context of transformation groups see [12, Chapter V.5.2, e.g. Theorem 3]. From the point of view of stochastics this means that moment conditions (roughly speaking the existence of a first moment of $X$ means differentiability of $\psi$ with respect to $u$ ) imply $t$-differentiability of $\psi$ and $\Phi$, and thus regularity. This has already been observed in [2]. However, moment conditions on the process $X$ are not natural in the present context of affine processes, and the question remained open, whether the moment conditions in [2] can simply be dropped. Finally let us mention that there is one wellknown case where moment conditions are not necessary, namely homogeneous affine processes, i.e., affine processes with $\psi(t, u)=u$ for $(t, u) \in \mathbb{R}_{\geq 0} \times i \mathbb{R}^{d}$. This is-up to killing of the process-precisely the case of Lévy processes. In this case $\Phi$ simply satisfies Cauchy's functional equation $\Phi(t+s, u)=\Phi(t, u) \Phi(s, u)$ and therefore regularity, that is differentiability of $\Phi$, follows by the classical result on (1.3).

We show in this article that even in the general case without moment conditions any stochastically continuous affine process is automatically regular. Hence the results of this paper truly extend [12] since no differentiability in $u$ is assumed. We introduce the basic definitions and some notation in Sect. 2 and show preliminary results in Sect. 3. In Sect. 4 we show regularity of an affine process subject to a condition of 'partial additivity'. Finally we show in Sect. 5 that all stochastically continuous affine processes defined on the domain $D=\mathbb{R}_{\geq 0}^{m} \times \mathbb{R}^{n}$ are regular by reducing the general question to regularity of the partially additive processes of Sect. 4. 


\section{Affine processes}

Definition 2.1 (Affine process) An affine process is a time-homogeneous Markov process $\left(X_{t}, \mathbb{P}^{x}\right)_{t \geq 0, x \in D}$ with state space $D=\mathbb{R}_{\geq 0}^{m} \times \mathbb{R}^{n}$, whose characteristic function is an exponentially-affine function of the state vector. This means that there exist functions $\Phi: \mathbb{R}_{\geq 0} \times i \mathbb{R}^{d} \rightarrow \mathbb{C}$ and $\psi: \mathbb{R}_{\geq 0} \times i \mathbb{R}^{d} \rightarrow \mathbb{C}^{d}$ such that

$$
\mathbb{E}^{x}\left[e^{\left\langle X_{t}, u\right\rangle}\right]=\Phi(t, u) \cdot \exp (\langle x, \psi(t, u)\rangle),
$$

for all $x \in D$, and for all $(t, u) \in \mathbb{R}_{\geq 0} \times i \mathbb{R}^{d}$.

Remark 2.2 The set $i \mathbb{R}^{d}$ denotes the purely imaginary numbers in $\mathbb{C}^{d}$, that is $i \mathbb{R}^{d}=$ $\left\{u \in \mathbb{C}^{d}: \operatorname{Re} u=0\right\}$.

Remark 2.3 The above definition differs in one detail from the definition given in [3]: In their article the right hand side of (2.1) is defined in terms of a function $\phi(t, u)$ as $\exp (\phi(t, u)+\langle x, \psi(t, u)\rangle)$, whereas we formulate the equation in terms of $\Phi(t, u)=\exp (\phi(t, u))$. In particular, we do not assume a priori that $\Phi(t, u) \neq 0$. The difference is subtle, but will play a role in Lemma 2.5 below, where we extend $\Phi(t, u)$ to a larger subset $\mathcal{Q}$ of the complex numbers. Note that our definition using $\Phi(t, u)$ is very close to that of [8].

Assumption 2.4 We will assume throughout this article that $X$ is stochastically continuous, i.e. for $t \rightarrow s$, the random variables $X_{t}$ converge to $X_{s}$ in probability, with respect to all $\left(\mathbb{P}^{x}\right)_{x \in D}$.

Note that the existence of a filtered space $\left(\Omega, \mathcal{F},\left(\mathcal{F}_{t}\right)_{t \geq 0}\right)$, where the process $\left(X_{t}\right)_{t \geq 0}$ is defined, is already implicit in the notion of a Markov process (we largely follow [13, Chapter III] in our notation and precise definition of a Markov process). $\mathbb{P}^{x}$ represents the law of the Markov process $\left(X_{t}\right)_{t \geq 0}$ started at $x$, i.e. we have that $X_{0}=x, \mathbb{P}^{x}$-almost surely.

Let us at this point introduce some additional notation: We write

$$
I=\{1, \ldots, m\} \quad \text { and } \quad J=\{m+1, \ldots, m+n\}
$$

for the index sets of the $\mathbb{R}_{\geq 0}^{m}$-valued component and the $\mathbb{R}^{n}$-valued component of $X$ respectively. For some vector $x \in \mathbb{R}^{d}$ we denote by $x=\left(x_{I}, x_{J}\right)$ its partition in the corresponding subvectors, and similarly for the function $\psi(t, u)=\left(\psi_{I}(t, u), \psi_{J}(t, u)\right)$. Also, $x_{i}$ denotes the $i$-th element of $x$, and $\left(e_{i}\right)_{i \in\{1, \ldots, d\}}$ are the unit vectors of $\mathbb{R}^{d}$. We will often write

$$
f_{u}(x):=\exp (\langle u, x\rangle)
$$

for the exponential function with $u \in \mathbb{C}^{d}$ and $x \in D$. A special role will be played by the set

$$
\mathcal{U}=\left\{u \in \mathbb{C}^{d}: \operatorname{Re} u_{I} \leq 0, \quad \operatorname{Re} u_{J}=0\right\}
$$


Note that $\mathcal{U}$ is precisely the set of all $u \in \mathbb{C}^{d}$, for which $x \mapsto f_{u}(x)$ is a bounded function on $D$. We also define

$$
\mathcal{U}^{\circ}=\left\{u \in \mathbb{C}^{d}: \operatorname{Re} u_{I}<0, \quad \operatorname{Re} u_{J}=0\right\} .
$$

Lemma 2.5 Let $\left(X_{t}\right)_{t \geq 0}$ be an affine process. Then

$$
\mathcal{Q}=\left\{(t, u) \in \mathbb{R}_{\geq 0} \times \mathcal{U}: \mathbb{E}^{0}\left[f_{u}\left(X_{t}\right)\right] \neq 0\right\},
$$

is open in $\mathbb{R}_{\geq 0} \times \mathcal{U}$ and there exists a unique continuous extension of $\Phi(t, u)$ and $\psi(t, u)$ to $\mathcal{Q}$, such that (2.1) holds for all $(t, u) \in \mathcal{Q}$. If $(t, u) \in\left(\mathbb{R}_{\geq 0} \times \mathcal{U}\right) \backslash \mathcal{Q}$, then $\mathbb{E}^{x}\left[f_{u}\left(X_{t}\right)\right]=0$ for all $x \in D$.

Remark 2.6 From the facts that $\{0\} \times \mathcal{U} \in \mathcal{Q}$ and that $\mathcal{Q}$ is open in $\mathbb{R}_{\geq 0} \times \mathcal{U}$ we can deduce the following: For every $u \in \mathcal{U}$ there exists $t_{*}(u)>0$, such that $(t, u) \in \mathcal{Q}$ for all $t \in\left[0, t_{*}(u)\right)$.

Proof We adapt the proof of [3, Lemma 3.1]: For $(t, u, x) \in \mathbb{R}_{\geq 0} \times \mathcal{U} \times D$ define $g(t, u, x)=\mathbb{E}^{x}\left[f_{u}\left(X_{t}\right)\right]$. We show that for fixed $x \in D$ the function $g(t, u, x)$ is jointly continuous in $(t, u)$ : Let $\left(t_{k}, u_{k}\right)$ be a sequence converging in $\mathcal{U}$ to $(t, u)$. By stochastic continuity of $X$ it holds that $X_{t_{k}} \rightarrow X_{t}$ in probability $\mathbb{P}^{x}$, and thus also in distribution. By dominated convergence we may therefore conclude that

$$
g\left(t_{k}, u_{k}, x\right)=\mathbb{E}^{x}\left[f_{u_{k}}\left(X_{t_{k}}\right)\right] \rightarrow \mathbb{E}^{x}\left[f_{u}\left(X_{t}\right)\right]=g(t, u, x),
$$

and thus that $g(t, u, x)$ is continuous in $(t, u)$. It follows that $\mathcal{Q}$ is open in $\mathbb{R}_{\geq 0} \times \mathcal{U}$. Because of the affine property (2.1) it holds that

$$
g(t, u, x) g(t, u, \xi)=g(t, u, x+\xi) g(t, u, 0)
$$

for all $(t, u) \in \mathbb{R}_{\geq 0} \times i \mathbb{R}^{d}$ and $x, \xi \in D$. But both sides of (2.5) are continuous functions of $u \in \overline{\mathcal{U}}$, and moreover analytic in $\mathcal{U}^{\circ}$. (This follows from well-known properties of the Laplace transform and the extension to its strip of regularity, cf. [3, Lemma A.2].) By the Schwarz reflection principle, (2.5) therefore holds for all $u \in \mathcal{U}$. Assume now that $(t, u) \in\left(\mathbb{R}_{\geq 0} \times \mathcal{U}\right) \backslash \mathcal{Q}$, such that $g(t, u, 0)=0$. Then it follows from (2.5) that $\mathbb{E}^{x}\left[f_{u}\left(X_{t}\right)\right]=g(t, u, x)=0$ for all $x \in D$, as claimed in the Lemma. On the other hand, for all $(t, u) \in \mathcal{Q}$ it holds that $\Phi(t, u) \neq 0$, such that we can define $h(x)=\Phi(t, u)^{-1} g(t, u, x)$. The function $h(x)$ is measurable and satisfies $h(x) h(\xi)=h(x+\xi)$ for all $x, \xi \in D$. Moreover $h(0) \neq 0$ by definition of $\mathcal{Q}$. Using a standard result on measurable solutions of the Cauchy equation (cf. [1, Sec. 2.2]) we conclude that there exists a unique continuous extension of $\psi(t, u)$ such that $\Phi(t, u)^{-1} g(t, u, x)=e^{\langle\psi(t, u), x\rangle}$, and the proof is complete.

From this point on, $\Phi(t, u)$ and $\psi(t, u)$ are defined on all of $\mathcal{Q}$, and given by the unique continuous extensions of Lemma 2.5. We can now give a precise definition of regularity of an affine process: 
Definition 2.7 An affine process $X$ is called regular, if the derivatives

$$
F(u)=\left.\frac{\partial}{\partial t} \Phi(t, u)\right|_{t=0}, \quad R(u)=\left.\frac{\partial}{\partial t} \psi(t, u)\right|_{t=0}
$$

exist and are continuous functions of $u \in \mathcal{U}$.

Remark 2.8 In [3] $F(u)$ is defined in a slightly different way, as the derivative of $\phi(t, u)$ at $t=0$. However the definitions are equivalent, since $\Phi(t, u)=\exp (\phi(t, u))$ and thus

$$
\left.\frac{\partial}{\partial t} \Phi(t, u)\right|_{t=0}=\left.e^{\phi(0, u)} \cdot \frac{\partial}{\partial t} \phi(t, u)\right|_{t=0}=\left.\frac{\partial}{\partial t} \phi(t, u)\right|_{t=0} .
$$

Proposition 2.9 Let $X$ be a stochastically continuous affine process. The functions $\Phi$ and $\psi$ have the following properties:

(i) $\Phi$ maps $\mathcal{Q}$ to the unit disc $\{u \in \mathbb{C}:|u| \leq 1\}$.

(ii) $\psi$ maps $\mathcal{Q}$ to $\mathcal{U}$.

(iii) $\Phi(0, u)=1$ and $\psi(0, u)=u$ for all $u \in \mathcal{U}$.

(iv) $\Phi$ and $\psi$ enjoy the 'semi-flow property': Suppose that $t, s \geq 0$ and $(t+s, u) \in \mathcal{Q}$. Then also $(t, u) \in \mathcal{Q}$ and $(s, \psi(t, u)) \in \mathcal{Q}$, and it holds that

$$
\begin{aligned}
& \Phi(t+s, u)=\Phi(t, u) \cdot \Phi(s, \psi(t, u)), \\
& \psi(t+s, u)=\psi(s, \psi(t, u)) .
\end{aligned}
$$

(v) $\Phi$ and $\psi$ are jointly continuous on $\mathcal{Q}$.

(vi) With the remaining arguments fixed, $u_{I} \mapsto \Phi(t, u)$ and $u_{I} \mapsto \psi(t, u)$ are analytic functions in $\left\{u_{I}: \operatorname{Re} u_{I}<0 ;(t, u) \in \mathcal{Q}\right\}$.

(vii) Let $(t, u) \in \mathcal{Q}$ and $w \in \mathcal{U}$ with $\operatorname{Re} u \leq \operatorname{Re} w$. Then also $(t, \operatorname{Re} w) \in \mathcal{Q}$ and

$$
\begin{aligned}
|\Phi(t, u)| & \leq \Phi(t, \operatorname{Re} w) \\
\operatorname{Re} \psi(t, u) & \leq \psi(t, \operatorname{Re} w) .
\end{aligned}
$$

Proof Let $(t, u) \in \mathcal{Q}$. Clearly $\left|\mathbb{E}^{x}\left[f_{u}\left(X_{t}\right)\right]\right| \leq \mathbb{E}^{x}\left[\left|f_{u}\left(X_{t}\right)\right|\right] \leq 1$. On the other hand $\mathbb{E}^{x}\left[f_{u}\left(X_{t}\right)\right]=\Phi(t, u) f_{\psi(t, u)}(x)$ by Lemma 2.5. Since $\left\|f_{u}\right\|_{\infty} \leq 1$ if and only if $u \in \mathcal{U}$, we conclude that $|\Phi(t, u)| \leq 1$ and $\psi(t, u) \in \mathcal{U}$ for all $(t, u) \in \mathcal{Q}$ and have shown (i) and (ii). Assertion (iii) follows immediately from $\mathbb{E}^{x}\left[f_{u}\left(X_{0}\right)\right]=f_{u}(x)$. For (iv) suppose that $(t+s, u) \in \mathcal{Q}$, such that

$$
\mathbb{E}^{x}\left[f_{u}\left(X_{t+s}\right)\right]=\Phi(t+s, u) f_{\psi(t+s, u)}(x)
$$

by Lemma 2.5. Applying the law of iterated expectations and the Markov property of $X$ it holds that

$$
\mathbb{E}^{x}\left[f_{u}\left(X_{t+s}\right)\right]=\mathbb{E}^{x}\left[\mathbb{E}^{x}\left[f_{u}\left(X_{t+s}\right) \mid \mathcal{F}_{s}\right]\right]=\mathbb{E}^{x}\left[\mathbb{E}^{X_{s}}\left[f_{u}\left(X_{t}\right)\right]\right]
$$


If $(t, u) \notin \mathcal{Q}$ then the inner expectation (and consequently the whole expression) evaluates to 0 , which is a contradiction to the fact that $(t+s, u) \in \mathcal{Q}$. It follows that $\mathbb{E}^{X_{s}}\left[f_{u}\left(X_{t}\right)\right]=\Phi(t, u) f_{\psi(t, u)}\left(X_{s}\right)$. If $(s, \psi(t, u)) \notin \mathcal{Q}$ then the outer expectation in (2.9) evaluates to 0 , which is also a contradiction. Thus also $(s, \psi(t, u)) \in \mathcal{Q}$, as claimed, and we can write (2.9) as

$\mathbb{E}^{x}\left[f_{u}\left(X_{t+s}\right)\right]=\mathbb{E}^{x}\left[\Phi(t, u) f_{\psi(t, u)}\left(X_{s}\right)\right]=\Phi(s, u) \cdot \Phi(t, \psi(s, u)) f_{\psi(t, \psi(s, u))}(x)$,

for all $x \in D$. Comparing with (2.8) the semi-flow equations (2.7) follow. Assertions (v) and (vi) can be derived directly from the proof of Lemma 2.5. To show (vii) note that

$$
\left|\mathbb{E}^{x}\left[f_{u}\left(X_{t}\right)\right]\right| \leq \mathbb{E}^{x}\left[\left|f_{u}\left(X_{t}\right)\right|\right]=\mathbb{E}^{x}\left[f_{(\operatorname{Re} u)}\left(X_{t}\right)\right] \leq \mathbb{E}^{x}\left[f_{(\operatorname{Re} w)}\left(X_{t}\right)\right],
$$

for all $x \in D$. Since $(t, u)$ in $\mathcal{Q}$ this implies that also $(t, \operatorname{Re} w) \in \mathcal{Q}$. From the affine property (2.1) we deduce that

$$
|\Phi(t, u)| \cdot \exp (\langle x, \operatorname{Re} \psi(t, u)\rangle) \leq \Phi(t, \operatorname{Re} w) \cdot \exp (\langle x, \psi(t, \operatorname{Re} w)\rangle) .
$$

Inserting first $x=0$ and then $C e_{i}$ with $C>0$ arbitrarily large yields the assertion.

Finally we show one additional technical property concerning the existence of derivatives of $\Phi$ and $\psi$ with respect to $u$, on the set $\mathcal{U}^{\circ}$.

Lemma 2.10 Let $X$ be a stochastically continuous affine process. For $i \in I$, the derivatives

$$
\frac{\partial}{\partial u_{i}} \Phi(t, u), \quad \frac{\partial}{\partial u_{i}} \psi(t, u)
$$

exist and are continuous for $(t, u) \in\left(\mathbb{R}_{\geq 0} \times \mathcal{U}^{\circ}\right) \cap \mathcal{Q}$.

Proof Let $i \in I$ and let $K$ be a compact subset of $\mathcal{U}^{\circ}$. It holds that

$$
\left|\frac{\partial}{\partial u_{i}} \exp \left(\left\langle u, X_{t}\right\rangle\right)\right|=\left|X_{t}^{i}\right| \cdot \exp \left(\left\langle\operatorname{Re} u, X_{t}\right\rangle\right) \text {. }
$$

The right hand side is uniformly bounded for all $t \in \mathbb{R}_{\geq 0}, u \in K$, and thus in particular uniformly integrable. We may conclude that $\frac{\partial}{\partial u_{i}} \mathbb{E}^{x}\left[e^{\left\langle X_{t}, u\right\rangle}\right]$ exists and is a continuous function of $(t, u) \in \mathbb{R}_{\geq 0} \times K$ for any $x \in D$. If in addition $(t, u) \in \mathcal{Q}$, then Lemma 2.5 states that $\mathbb{E}^{x}\left[e^{\left\langle X_{t}, u\right\rangle}\right]=\Phi(t, u) \exp (\langle x, \psi(t, u)\rangle)$. Since $K$ was an arbitrary compact subset of $\mathcal{U}^{\circ}$ the claim follows.

\section{Affine processes are Feller processes}

In this section we prove the Feller property for all affine processes. For regular affine processes, this has been shown in [3]; here we give a proof that does not require a regularity assumption. The key to the proof are the following properties of the function 
$\psi(t, u)$ for a given stochastically continuous affine process $X$, which will also be used in the proof of our main result in Sect. 5:

Property A: $\psi(t,$.$) maps \mathcal{U}^{\circ}$ to $\mathcal{U}^{\circ}$.

Property B: $\psi_{J}(t, u)=e^{\beta t} u_{J}$ for all $(t, u) \in \mathcal{Q}$, with $\beta$ a real $n \times n$-matrix.

Let us give here an intuitive example illustrating the second property, which has already been observed in [2, Prop 2.1 and Cor. 2.1]: Consider an affine process with onedimensional state space $D=\mathbb{R}$, and the property that $\Phi(t, u)=1$. Then for any initial value $x \in \mathbb{R}$

$$
\mathbb{E}^{x}\left[f_{i y}\left(X_{t}\right)\right]=e^{x \psi(t, i y)} \text { and } \mathbb{E}^{-x}\left[f_{i y}\left(X_{t}\right)\right]=e^{-x \psi(t, i y)}
$$

are both characteristic functions, and moreover reciprocal to each other. But a wellknown result (cf. [11, Thm. 2.1.4]) states that the only characteristic functions, whose reciprocals are also characteristic functions correspond to degenerate distributions, i.e. Dirac measures. Here, this implies that $\psi(t, i y)=i y m(t)$, for $m(t)$ a deterministic function. Moreover, by the Markov property $m(t+s)=m(t) m(s)$, which is Cauchy's functional equation with the unique continuous solution $m(t)=e^{\lambda t} m(0)$, for some $\lambda \in \mathbb{R}$. Hence, $\psi(t, i y)$ is necessarily of the form $e^{\lambda t} i y$ and satisfies therefore Property $B$.

As we shall show, the argument can be extended to the case of arbitrary $\Phi(t, u)$ and to the general state space $D=\mathbb{R}_{\geq 0}^{m} \times \mathbb{R}^{n}$. The next Lemma is the first step in this direction:

Lemma 3.1 Let $X$ be a stochastically continuous affine process. Let $K \subseteq\{1, \ldots, d\}$, $k \in\{1, \ldots, d\}$, and let $\left(t_{n}\right)_{n \in \mathbb{N}}$ be a sequence such that $t_{n} \downarrow 0$. Define $\Omega_{K}:=$ $\left\{y \in \mathbb{R}^{d}: y_{i}=0\right.$ for $\left.i \notin K\right\}$, and suppose that

$$
\operatorname{Re} \psi_{k}\left(t_{n}, i y\right)=0 \text { for all } y \in \Omega_{K} \text { and } n \in \mathbb{N} \text {. }
$$

Then there exist $\zeta_{k}\left(t_{n}\right) \in \mathbb{R}^{|K|}$ and an increasing sequence of positive numbers $R_{n}$ such that $R_{n} \uparrow \infty$ and

$$
\psi_{k}\left(t_{n}, i y\right)=\left\langle\zeta_{k}\left(t_{n}\right), i y_{K}\right\rangle
$$

for all $y \in \Omega_{K}$ with $|y|<R_{n}$.

For the proof we will use the following result:

Lemma 3.2 Let $\Theta$ be a positive definite function on $\mathbb{R}^{d}$ with $\Theta(0)=1$. Then

$$
|\Theta(y+z)-\Theta(y) \Theta(z)|^{2} \leq\left(1-|\Theta(y)|^{2}\right)\left(1-|\Theta(z)|^{2}\right) \leq 1
$$

for all $y, z \in \mathbb{R}^{d}$. 
Proof of Lemma 3.2 The result follows from considering the matrix

$$
M_{\Theta}(y, z):=\left(\begin{array}{ccc}
\Theta(0) & \overline{\Theta(y)} & \Theta(z) \\
\Theta \overline{(y)} & \Theta(0) & \Theta(y+z) \\
\hline \Theta(z) & \frac{\Theta(y+z)}{\Theta(0)} & \Theta(0)
\end{array}\right), \quad y, z \in \mathbb{R}^{d}, y \neq z,
$$

which is positive semi-definite by definition of $\Theta$. The inequality is then derived from the fact that det $M_{\Theta}(y, z) \geq 0$. See [7, Lemma 3.5.10] for details.

Proof of Lemma 3.1 As the characteristic function of the (possibly defective) random variable $X_{t_{n}}$ under $\mathbb{P}^{x}$, the function $y \mapsto \mathbb{E}^{x}\left[f_{i y}\left(X_{t_{n}}\right)\right]$ is positive definite for any $x \in D, n \in \mathbb{N}$. We define now for every $y \in \Omega_{K}, c>0$, and $n \in \mathbb{N}$, the function

$$
\Theta(y ; n, c):=\frac{1}{\Phi\left(t_{n}, 0\right)} \mathbb{E}^{c e_{k}}\left[f_{i y}\left(X_{t_{n}}\right)\right]=\frac{\Phi\left(t_{n}, i y\right)}{\Phi\left(t_{n}, 0\right)} \exp \left(c \cdot \psi_{k}\left(t_{n}, i y\right)\right) .
$$

Clearly, as a function of $y \in \Omega_{K}$, also $\Theta(y ; n, c)$ is positive definite. In addition it satisfies $\Theta(0 ; n, c)=\exp \left(c \cdot \psi_{k}\left(t_{n}, 0\right)\right)=1$, for large enough $n$, say $n \geq N$, by the following argument: It should be obvious, that $\exp \left(c \cdot \psi_{k}\left(t_{n}, 0\right)\right)$ is always a real quantity. By assumption, $\psi_{k}\left(t_{n}, 0\right)$ is purely imaginary, such that it must be an integer multiple of $\pi$ for all $n \in \mathbb{N}$. But $\psi_{k}(0,0)=0$, and $\psi_{k}(t, 0)$ is continuous in $t$ by Proposition 2.9, and we conclude $\psi_{k}\left(t_{n}, 0\right)=0$ for large enough $n$.

Thus, for $n \geq N$, we may apply Lemma 3.2 to $\Theta$, and conclude that for any $y, z \in \Omega_{K}, c>0$ and $n \geq N$

$$
|\Theta(y+z ; n, c)-\Theta(y ; n, c) \cdot \Theta(z ; n, c)|^{2} \leq 1 .
$$

For compact notation we define the abbreviations

$$
\begin{aligned}
r_{1} & =\left|\frac{\Phi\left(t_{n}, i(y+z)\right)}{\Phi\left(t_{n}, 0\right)}\right|, \quad r_{2}=\left|\frac{\Phi\left(t_{n}, i y\right) \Phi\left(t_{n}, i z\right)}{\Phi\left(t_{n}, 0\right)^{2}}\right|, \\
\alpha_{1} & =\operatorname{Arg} \frac{\Phi\left(t_{n}, i(y+z)\right)}{\Phi\left(t_{n}, 0\right)}, \quad \alpha_{2}=\operatorname{Arg} \frac{\Phi\left(t_{n}, i y\right) \Phi\left(t_{n}, i z\right)}{\Phi\left(t_{n}, 0\right)^{2}}, \\
\beta_{1} & =\operatorname{Im} \psi_{k}\left(t_{n}, i(y+z)\right), \quad \beta_{2}=\operatorname{Im} \psi_{k}\left(t_{n}, i y\right)+\operatorname{Im} \psi_{k}\left(t_{n}, i z\right),
\end{aligned}
$$

where we suppress the dependency on $y, t, z$ for the moment. It holds that

$$
\left|r_{1} e^{\left(\alpha_{1}+c \beta_{1}\right) i}-r_{2} e^{\left(\alpha_{2}+c \beta_{2}\right) i}\right|^{2}=r_{1}^{2}+r_{2}^{2}-2 r_{1} r_{2} \cos \left(\alpha_{1}-\alpha_{2}+\left(\beta_{1}-\beta_{2}\right) c\right) .
$$

Using the elementary inequality $2 r_{1} r_{2} \leq r_{1}^{2}+r_{2}^{2}$, we derive

$$
2 r_{1} r_{2}\left\{1-\cos \left(\alpha_{1}-\alpha_{2}+\left(\beta_{1}-\beta_{2}\right) c\right)\right\} \leq\left|r_{1} e^{\left(\alpha_{1}+c \beta_{1}\right) i}-r_{2} e^{\left(\alpha_{2}+c \beta_{2}\right) i}\right|^{2}
$$


which combined with inequality (3.1) yields

$$
r_{1} r_{2}\left(1-\cos \left(\alpha_{1}-\alpha_{2}+\left(\beta_{1}-\beta_{2}\right) c\right)\right) \leq \frac{1}{2}
$$

Define now $R_{n}=0$ for $n<N$, and

$$
R_{n}:=\sup \left\{\rho \geq 0: r_{1}\left(t_{n}, y, z\right) r_{2}\left(t_{n}, y, z\right)>\frac{1}{2} \text { for } y, z \in \Omega_{K} \text { with }|y| \leq \rho,|z| \leq \rho\right\}
$$

for $n \geq N$. Note that $R_{n} \uparrow \infty$ : This follows from $r_{1}(0, y, z)=r_{2}(0, y, z)=1$ for all $y, z \in \Omega_{K}$, and the continuity of $r_{1}$ and $r_{2}$.

Suppose that

$$
\beta_{1}-\beta_{2}=\operatorname{Im} \psi_{k}\left(t_{n}, i(y+z)\right)-\operatorname{Im} \psi_{k}\left(t_{n}, i y\right)-\operatorname{Im} \psi_{k}\left(t_{n}, i z\right) \neq 0
$$

for any $n \in \mathbb{N}$ and $y, z \in \Omega_{K}$ with $|y|<R_{n},|z|<R_{n}$. Then there exists an $c>0$ such that

$$
\cos \left(\alpha_{1}-\alpha_{2}+\left(\beta_{1}-\beta_{2}\right) c\right)=-1
$$

Inserting into (3.2) we obtain

$$
\frac{1}{2} \cdot 2<r_{1} r_{2}\left(1-\cos \left(\alpha_{1}-\alpha_{2}+\left(\beta_{1}-\beta_{2}\right) c\right)\right) \leq \frac{1}{2},
$$

a contradiction. We conclude that

$$
\beta_{1}-\beta_{2}=\operatorname{Im} \psi_{k}\left(t_{n}, i(y+z)\right)-\operatorname{Im} \psi_{k}\left(t_{n}, i y\right)-\operatorname{Im} \psi_{k}\left(t_{n}, i z\right)=0,
$$

for all $y, z \in \Omega_{K}$ with $|y|<R_{n},|z|<R_{n}$. Equation (3.3) is nothing but Cauchy's first functional equation. Since $\psi(t,$.$) is continuous, it follows that \operatorname{Im} \psi_{k}$ is a linear function of $y_{K}$. In addition, $\operatorname{Re} \psi_{k}\left(t_{n}, y\right)$ is zero, by assumption, such that there exists some real vector $\zeta_{k}\left(t_{n}\right)$ with

$$
\psi_{k}\left(t_{n}, i y\right)=\left\langle\zeta_{k}\left(t_{n}\right), i y_{K}\right\rangle
$$

for all $y \in \Omega_{K}$ with $|y|<R_{n}$, and the Lemma is proved.

We use the above Lemma to show the following Proposition, which implies Property $\mathrm{B}$ of $\psi$, that was introduced at the beginning of the section:

Proposition 3.3 Let $\left(X_{t}\right)_{t \geq 0}$ be a stochastically continuous affine process on $D=$ $\mathbb{R}_{\geq 0}^{m} \times \mathbb{R}^{n}$ and denote by $J$ its real-valued components. Then there exists a real $n \times n-$ matrix $\beta$ such that $\psi_{J}(t, u)=e^{t \beta} u_{J}$ for all $(t, u) \in \mathcal{Q}$. 
Proof Consider the definition of $\mathcal{U}$ in (2.2). Since $\psi(t, u)$ takes by Proposition 2.9 values in $\mathcal{U}$ it is clear that $\operatorname{Re} \psi_{J}(t, i y)=0$ for any $(t, y) \in \mathbb{R}_{\geq 0} \times \mathbb{R}^{d}$. Fix now some $t_{*}>0$ and define $t_{n}:=t_{*} / n$ for all $n \in \mathbb{N}$. We can apply Lemma 3.1 with $K=\{1, \ldots, d\}$ and any choice of $k \in J$, to obtain a sequence $R_{n} \uparrow \infty$ (even independent of $k$ ), such that

$$
\psi_{J}\left(t_{n}, i y\right)=\Xi\left(t_{n}\right) \cdot i y,
$$

for all $y \in \mathbb{R}^{d}$ with $|y|<R_{n}$. Here $\Xi\left(t_{n}\right)$ denotes the real $n \times d$-matrix formed by concatenating the column vectors $\left(\zeta_{k}\left(t_{n}\right)\right)_{k=1, \ldots, d}$ obtained from Lemma 3.1.

Let $i \in I, n \in \mathbb{N}$, define $\Omega_{n}:=\left\{\omega \in \mathbb{C}:|\omega| \leq R_{n},\left(t_{n}, e_{i} \omega\right) \in \mathcal{Q}\right\}$, and consider the function

$$
h_{n}: \Omega_{n} \rightarrow \mathbb{C}^{n}: \quad \omega \mapsto \psi_{J}\left(t_{n}, \omega e_{i}\right)-\Xi(t) \cdot \omega e_{i} .
$$

This is an analytic function on $\Omega_{n}^{\circ}$ and continuous on $\Omega_{n}$. According to the Schwarz reflection principle, $h_{n}$ can be extended to an analytic function on an open superset of $\Omega_{n}$. But (3.5) implies that the function $h_{n}$ takes the value 0 on a subset with an accumulation point in $\mathbb{C}$. We conclude that $h_{n}$ is zero everywhere. In particular we have that

$$
0=\operatorname{Re} \psi_{J}\left(t_{n}, \omega e_{i}\right)-\Xi\left(t_{n}\right) \cdot \operatorname{Re} \omega e_{i}=\Xi\left(t_{n}\right) \cdot \operatorname{Re} \omega e_{i},
$$

for all $\omega \in \Omega_{n}$. This can only hold true, if the $i$-th column of $\Xi\left(t_{n}\right)$ is zero. Since $i \in I$ was arbitrary we have reduced (3.5) to

$$
\psi_{J}\left(t_{n}, u\right)=\Xi_{0}\left(t_{n}\right) \cdot u_{J}
$$

for all $\left(t_{n}, u\right) \in \mathcal{Q}$, such that $\left|u_{J}\right|<R_{n}$. Here $\Xi_{0}\left(t_{n}\right)$ denotes the $n \times n$-submatrix of $\Xi\left(t_{n}\right)$ that results from dropping the zero-columns.

Fix an arbitrary $u_{*} \in \mathcal{U}$ with $\left(t_{*}, u_{*}\right) \in \mathcal{Q}$. By Proposition 2.9 we know that also $\left(t, u_{*}\right) \in \mathcal{Q}$ for all $t \in\left[0, t_{*}\right]$, such that $R:=\sup \left\{\left|\psi_{K}\left(t, u_{*}\right)\right|: t \in\left[0, t_{*}\right]\right\}$ is welldefined. Since $\psi(t, u)$ is continuous, $R$ is finite. Choose $N$ such that $R_{n}>R$ for all $n \geq N$. Using the semi-flow equation we can write $\psi_{J}\left(t_{*}, u_{*}\right)$ as

$$
\begin{aligned}
\psi_{J}\left(t_{*}, u_{*}\right) & =\psi_{J}\left(t_{n}, \psi\left(t_{*} \frac{n-1}{n}, u_{*}\right)\right) \\
& =\Xi_{0}\left(t_{n}\right) \cdot \psi_{J}\left(t_{*} \frac{n-1}{n}, u_{*}\right)=\cdots=\Xi_{0}\left(t_{n}\right)^{n} \cdot u_{*} ;
\end{aligned}
$$

for any $n \geq N$. Thus, the functional equation $\psi(t, u)=\Xi_{0}(t) \cdot u_{J}$ actually holds for all $(t, u) \in \mathcal{Q}$. Another application of the semi-flow property yields then, that

$$
\Xi_{0}(t+s)=\Xi_{0}(t) \Xi_{0}(s), \text { for all } t, s \geq 0 .
$$

Since $\Xi_{0}(0)=1, \Xi_{0}$ is continuous and satisfies the second Cauchy functional equation, it follows that $\Xi_{0}(t)=e^{\beta t}$ for some real $n \times n$-matrix $\beta$, which completes the proof. 
The next proposition shows that also Property A holds true for $\psi$, as we have claimed at the beginning of the section.

Proposition 3.4 Suppose that $(t, u) \in \mathcal{Q}$. If $u \in \mathcal{U}^{\circ}$, then $\psi(t, u) \in \mathcal{U}^{\circ}$.

Proof For a contradiction, assume there exists $(t, u) \in \mathcal{Q}$ such that $u \in \mathcal{U}^{\circ}$, but $\psi(t, u) \notin \mathcal{U}^{\circ}$. This implies that there exists $k \in I$, such that $\operatorname{Re} \psi_{k}(t, u)=0$. Let $\mathcal{Q}_{t, k}=\left\{\omega \in \mathbb{C}: \operatorname{Re} \omega \leq 0 ;\left(t, \omega e_{k}\right) \in \mathcal{Q}\right\}$. From the inequalities of Proposition 2.9.vii we deduce that

$$
0=\operatorname{Re} \psi_{k}(t, u) \leq \psi_{k}\left(t, \operatorname{Re} \omega \cdot e_{k}\right) \leq 0,
$$

and thus that $\psi_{k}\left(t, \operatorname{Re} \omega \cdot e_{k}\right)=0$ for all $\omega \in \mathcal{Q}_{t, k}$ with $\operatorname{Re} u_{k} \leq \operatorname{Re} \omega$. By Proposition 2.9(vi), $\psi_{k}\left(t, \omega e_{k}\right)$ is an analytic function of $\omega$. Since it takes the value zero on a set with an accumulation point, it is zero everywhere, i.e. $\psi_{k}\left(t, \omega e_{k}\right)=0$ for all $\omega \in \mathcal{Q}_{t, k}$. The same statement holds true for $t$ replaced by $t / 2: \operatorname{Set} \lambda:=\operatorname{Re} \psi_{k}(t / 2, u)$. If $\lambda=0$, we can proceed exactly as above, only with $t / 2$ instead of $t$. If $\lambda<0$, then we have, by another application of Proposition 2.9(vii), that

$0=\operatorname{Re} \psi_{k}(t, u)=\operatorname{Re} \psi_{k}(t / 2, \psi(t / 2, u)) \leq \psi_{k}\left(t / 2, \lambda e_{k}\right) \leq \psi_{k}\left(t / 2, \operatorname{Re} \omega e_{k}\right) \leq 0$,

for all $\omega \in \mathcal{Q}_{t / 2, k}$ such that $\lambda \leq \operatorname{Re} \omega$. Again we use that an analytic function that takes the value zero on a set with accumulation point, is zero everywhere, and obtain that $\psi_{k}\left(t / 2, \omega e_{k}\right)=0$ for all $\omega \in \mathcal{Q}_{t / 2, k}$. Repeating this argument, we finally obtain a sequence $t_{n} \downarrow 0$, such that

$$
\psi_{k}\left(t_{n}, \omega e_{k}\right)=0 \text { for all } \omega \in \mathcal{Q}_{t_{n}, k}
$$

We can now apply Lemma 3.1 with $K=\{k\}$, which implies that $\psi_{k}$ is of the linear form

$$
\psi_{k}\left(t_{n}, \omega e_{k}\right)=\zeta_{k}\left(t_{n}\right) \cdot \omega, \quad \text { for all } \omega \in \mathcal{Q}_{t_{n}, k} \text { with }|\omega| \leq R_{n}
$$

where $\zeta_{k}\left(t_{n}\right)$ are real numbers, and $R_{n} \uparrow \infty$. Note that since $\zeta_{k}\left(t_{n}\right) \rightarrow 1$ as $t_{n} \rightarrow 0$, we have that $\zeta_{k}\left(t_{n}\right)>0$ for $n$ large enough. Choosing now some $\omega_{*}$ with $\operatorname{Re} \omega_{*}<0$ it follows that $\operatorname{Re} \psi_{k}\left(t_{n}, \omega_{*} e_{k}\right)<0$-with strict inequality. This is a contradiction to (3.9), and the assertion is shown.

We are now prepared to show the main result of this section:

Theorem 3.5 Every stochastically continuous affine process $X$ is a Feller process.

Remark 3.6 As an immediate Corollary to this theorem, every stochastically continuous affine process has a càdlàg version, see for instance [13].

Proof By stochastic continuity of $\left(X_{t}\right)_{t \geq 0}$ and dominated convergence, it follows immediately that $P_{t} f(x)=\mathbb{E}^{x}\left[f\left(X_{t}\right)\right] \rightarrow f(x)$ as $t \rightarrow 0$ for all $f \in C_{0}(D)$ and $x \in D$. To prove the Feller property of $\left(X_{t}\right)_{t \geq 0}$ it remains to show that 
$P_{t}\left(C_{0}(D)\right) \subseteq C_{0}(D)$ : For $u_{I} \in \mathbb{C}^{m}$ with $\operatorname{Re} u_{I}<0$ and $g \in C_{c}^{\infty}\left(\mathbb{R}^{n}\right)$, i.e. a smooth function with compact support, define the functions

$$
h\left(x ; u_{I}, g\right)=e^{\left\langle u_{I}, x_{I}\right\rangle} \int_{\mathbb{R}^{n}} f_{i y}\left(x_{J}\right) g(y) d y
$$

mapping $D$ to $\mathbb{C}$, and the set

$$
P:=\left\{h\left(x ; u_{I}, g\right): u_{I} \in \mathbb{C}^{d}, \operatorname{Re} u_{I}<0, g \in C_{c}^{\infty}\left(\mathbb{R}^{n}\right)\right\}
$$

Denote by $\mathcal{L}(P)$ the set of (complex) linear combinations of functions in $P$. From the Riemann-Lebesgue Lemma it follows that $\int_{\mathbb{R}^{n}} f_{i y}\left(x_{J}\right) g(y) d y$ vanishes at infinity, and thus that $\mathcal{L}(P) \subset C_{0}(D)$. It is easy to see that $\mathcal{L}(P)$ is a subalgebra of $C_{0}(D)$, that is in addition closed under complex conjugation and multiplication. (Note that the product of two Fourier transforms of compactly supported functions $g_{1}, g_{2}$ is the Fourier transform of a compactly supported function, namely $g_{1} * g_{2}$.) It is also straight-forward to check that $\mathcal{L}(P)$ is point separating and vanishes nowhere (i.e. there is no $x_{0} \in D$ such that $h\left(x_{0}\right)=0$ for all $\left.h \in \mathcal{L}(P)\right)$. Using a suitable version of the Stone-Weierstrass theorem (e.g. [14, Corollary 7.3.9]), it follows that $\mathcal{L}(P)$ is dense in $C_{0}(D)$.

Fix some $t \in \mathbb{R}_{\geq 0}$ and let $h(x) \in P$. By Lemma 2.5 it holds that $\mathbb{E}^{x}\left[f_{u}\left(X_{t}\right)\right]=$ $\Phi(t, u) \exp (\langle x, \psi(t, u)\rangle)$ whenever $(t, u) \in \mathcal{Q}$, and $\mathbb{E}^{x}\left[f_{u}\left(X_{t}\right)\right]=0$ whenever $(t, u) \notin \mathcal{Q}$. Moreover, by Proposition 3.3 we know that $\psi_{J}(t, u)=e^{\beta t} u_{J}$ for all $(t, u) \in \mathcal{Q}$. Thus, writing $u=\left(u_{I}, i y\right)$, we have

$$
\begin{aligned}
P_{t} h(x)= & \mathbb{E}^{x}\left[\int_{\mathbb{R}^{n}} f_{\left(u_{I}, i y\right)}\left(X_{t}\right) g(y) d y\right]=\int_{\mathbb{R}^{n}} \mathbb{E}^{x}\left[f_{\left(u_{I}, i y\right)}\left(X_{t}\right)\right] g(y) d y \\
= & \int_{\{u \in \mathcal{U}:(t, u) \in \mathcal{Q}\}} \mathbb{E}^{x}\left[f_{\left(u_{I}, i y\right)}\left(X_{t}\right)\right] g(y) d y \\
= & \int_{\{u \in \mathcal{U}:(t, u) \in \mathcal{Q}\}} \Phi\left(t, u_{I}, i y\right) \exp \left(\left\langle x_{I}, \psi_{I}\left(t, u_{I}, i y\right)\right\rangle+\left\langle x_{J}, e^{t \beta} i y\right\rangle\right) g(y) d y .
\end{aligned}
$$

Since $\left(u_{I}, i y\right) \in \mathcal{U}^{\circ}$ it follows by Proposition 3.4 that also $\operatorname{Re} \psi_{I}\left(t, u_{I}, i y\right)<0$ for any $y \in \mathbb{R}^{n}$. This shows that $P_{t} h(x) \rightarrow 0$ as $\left|x_{I}\right| \rightarrow \infty$. In addition, as a function of $x_{J}$, (3.10) can be interpreted as the Fourier transformation of a compactly supported density. The Riemann-Lebesgue Lemma then implies that $P_{t} h(x) \rightarrow 0$ as $\left|x_{J}\right| \rightarrow \infty$, and we conclude that $P_{t} h \in C_{0}(D)$. The assertion extends by linearity to every $h \in \mathcal{L}(P)$, and finally by the density of $\mathcal{L}(P)$ to every $h \in C_{0}(D)$. This proves that the semi-group $\left(P_{t}\right)_{t \geq 0}$ maps $C_{0}(D)$ into $C_{0}(D)$, and hence that $\left(X_{t}\right)_{t \geq 0}$ is a Feller process. 


\section{All partially additive affine processes are regular}

Definition 4.1 We say that a stochastically continuous affine process is partially additive, if for all $x \in D,(t, u) \in \mathbb{R}_{\geq 0} \times i \mathbb{R}^{d}$

$$
\mathbb{E}^{x}\left[e^{\left\langle X_{t}, u\right\rangle}\right]=e^{\left\langle x_{J}, u_{J}\right\rangle} \cdot \mathbb{E}^{\left(x_{I}, 0\right)}\left[e^{\left\langle X_{t}, u\right\rangle}\right]
$$

The above condition is equivalent to the statement that for any $y$ of the form $y=\left(0, y_{J}\right)$, the law of $X_{t}+y$ under $\mathbb{P}^{x}$ equals the law of $X_{t}$ under $\mathbb{P}^{(x+y)}$. If this held true for any $y \in D$ the process would be a (completely) additive timehomogeneous Markov process, i.e. a Lévy process, possibly killed at an exponential rate. Since we impose the condition only for $y$ of the form $\left(0, y_{J}\right)$, we call the process partially additive. Note that partially additive affine processes are frequently encountered in mathematical finance: Affine stochastic volatility models (e.g. the Heston model) typically have the partial additivity property; see [9].

Combining Definition 4.1 with the affine property (2.1), it is easy to see that the following holds:

Lemma 4.2 A stochastically continuous affine process is partially additive, if and only if $\psi_{J}(t, u)=u_{J}$ for all $(t, u) \in \mathbb{R}_{\geq 0} \times i \mathbb{R}^{d}$ (or equivalently for all $(t, u) \in \mathcal{Q}$ ).

The main result of this section is the following:

Theorem 4.3 Every partially additive, stochastically continuous affine process is regular.

Our proof uses the techniques originally presented in [12] for continuous transformation groups, and follows in part the proof of [2, Theorem 4.1].

Proof To simplify calculations we embed $\Phi(t, u)$ and $\psi(t, u)$ into the extended semiflow $\Upsilon(t, u)$, that is we set $\widehat{\mathcal{Q}}:=\mathcal{Q} \times \mathbb{C}$ and define

$$
\Upsilon: \widehat{\mathcal{Q}} \rightarrow \mathbb{C}^{d+1}, \quad\left(t, u_{1}, \ldots u_{d}, u_{d+1}\right) \mapsto\left(\begin{array}{c}
\psi\left(t,\left(u_{1}, \ldots, u_{d}\right)\right) \\
\Phi\left(t,\left(u_{1}, \ldots, u_{d}\right)\right) \cdot u_{d+1}
\end{array}\right) .
$$

Note that all vectors $u$ have now a $(d+1)$ th component added; this component will be assigned to the non-negative components $I$, such that under slight abuse of notation we now write $I=\{1, \ldots, m, d+1\}$. The semi-flow property is preserved by $\Upsilon(t, u)$, i.e. $\Upsilon(t+s, u)=\Upsilon(t, \Upsilon(s, u))$ for all $(t+s, u) \in \widehat{\mathcal{Q}}$. The partial additivity condition on $X$ implies that $\Upsilon_{J}(t, u)=u_{J}$ for all $(t, u) \in \widehat{\mathcal{Q}}$. Clearly, the time derivative exists and vanishes, i.e.,

$$
\left.\frac{\partial}{\partial t} \Upsilon_{J}(t, u)\right|_{t=0}=0
$$

for all $u \in \mathcal{U} \times \mathbb{C}$. In the rest of the proof we thus focus on the remaining (nonadditive) components. Let $u \in \widehat{\mathcal{U}}^{\circ}:=\mathcal{U}^{\circ} \times \mathbb{C}$ be fixed and assume that $t, s \in \mathbb{R}_{\geq 0}$ 
are small enough such that $\Phi(t+s, u), \psi(t+s, u)$ and their $u$-derivatives are always well-defined (cf. Lemma 2.10). Denote by $\frac{\partial \Upsilon_{I}}{\partial u_{I}}(t, u)$ the Jacobian of $\Upsilon_{I}$ with respect to $u_{I}$. Using a Taylor expansion we have that

$$
\begin{aligned}
\int_{0}^{s} \Upsilon_{I}(r, \Upsilon(t, u)) d r-\int_{0}^{s} \Upsilon_{I}(t, u) d r= & \int_{0}^{s} \frac{\partial \Upsilon_{I}}{\partial u_{I}}(r, u) d r \cdot\left(\Upsilon_{I}(t, u)-u_{I}\right) \\
& +o\left(\left\|\Upsilon_{I}(t, u)-u_{I}\right\|\right)
\end{aligned}
$$

On the other hand, using the semi-flow property of $\Upsilon$ we can write the left side of (4.3) as

$$
\begin{aligned}
\int_{0}^{s} \Upsilon_{I}(r, \Upsilon(t, u)) d r-\int_{0}^{s} \Upsilon_{I}(r, u) d r & =\int_{0}^{s} \Upsilon_{I}(r+t, u) d r-\int_{0}^{s} \Upsilon_{I}(r, u) d r \\
& =\int_{t}^{s+t} \Upsilon_{I}(r, u) d r-\int_{0}^{s} \Upsilon_{I}(r, u) d r \\
& =\int_{s}^{s+t} \Upsilon_{I}(r, u) d r-\int_{0}^{t} \Upsilon_{I}(r, u) d r \\
& =\int_{0}^{t} \Upsilon_{I}(r+s, u) d r-\int_{0}^{t} \Upsilon_{I}(r, u) d r
\end{aligned}
$$

Denoting the last expression by $I(s, t)$ and combining (4.3) with (4.4) we obtain

$$
\lim _{t \downarrow 0} \frac{\left\|\frac{1}{s} I(s, t)\right\|}{\left\|\Upsilon_{I}(t, u)-u_{I}\right\|}=\left\|\frac{1}{s} \int_{0}^{s} \frac{\partial \Upsilon_{I}}{\partial u_{I}}(r, u) d r\right\| .
$$

Define $M(s, u):=\frac{1}{s} \int_{0}^{s} \frac{\partial \Upsilon_{I}}{\partial u_{I}}(r, u) d r$. Note that as $s \rightarrow 0$, it holds that $M(s, u) \rightarrow$ $\frac{\partial \Upsilon_{I}}{\partial u_{I}}(0, u)=I_{I}$ (the identity matrix). Thus for $s$ small enough $\|M(s, u)\| \neq 0$, and we conclude that

$$
\begin{aligned}
& \lim _{t \downarrow 0} \frac{1}{t}\left\|\Upsilon_{I}(t, u)-u_{I}\right\| \\
& \quad=\left\|\lim _{t \downarrow 0} \frac{I(s, t)}{s t}\right\| \cdot\|M(s, u)\|^{-1}=\left\|\frac{\Upsilon_{I}(s, u)-u_{I}}{s}\right\| \cdot\|M(s, u)\|^{-1} .
\end{aligned}
$$

The right hand side of (4.5) is well-defined and finite, implying that also the limit on the left hand side is. Thus, combining (4.3) and (4.4), dividing by st and taking the 
limit $t \downarrow 0$ we obtain

$$
\lim _{t \downarrow 0} \frac{\Upsilon_{I}(t, u)-u_{I}}{t}=\frac{\Upsilon_{I}(s, u)-u_{I}}{s} \cdot M(s, u)^{-1} .
$$

Again we may choose $s$ small enough, such that $M(s, u)$ is invertible, and the right hand side of the above expression is well-defined. The existence and finiteness of the right hand side then implies the existence of the limit on the left. In addition the right hand side is a continuous function of $u \in \widehat{\mathcal{U}}^{\circ}$, such that also the left hand side is. Adding back the components $J$, for which a time derivative trivially exists (recall that $\psi_{J}(t, u)=u_{J}$ for all $\left.t \geq 0\right)$, we obtain that

$$
\mathcal{R}(u):=\lim _{t \downarrow 0} \frac{\Upsilon(t, u)-u}{t}=\left.\frac{\partial}{\partial t} \Upsilon(t, u)\right|_{t=0}
$$

exists and is a continuous function of $u \in \widehat{\mathcal{U}}^{\circ}$. Denoting the first $d$ components of $\mathcal{R}(u)$ by $R(u)$ and the $d+1$ th component by $F(u)$ we can 'disentangle' the extended semi-flow $\Upsilon$, drop the $(d+1)$ th component of $u$, and see that

$$
F(u):=\left.\frac{\partial}{\partial t} \Phi(t, u)\right|_{t=0} \text { and } \quad R(u):=\left.\frac{\partial}{\partial t} \psi(t, u)\right|_{t=0}
$$

are likewise well-defined and continuous on $\mathcal{U}^{\circ}$.

To show that $\left(X_{t}\right)_{t \geq 0}$ is regular affine it remains to show that (4.7) extends continuously to $\mathcal{U}$ : To this end let $t_{n} \downarrow 0, x \in D, u \in \mathcal{U}^{\circ}$, and rewrite (4.7) as

$$
\begin{aligned}
F(u)+\langle x, R(u)\rangle & =\lim _{n \rightarrow \infty} \frac{\Phi\left(t_{n}, u\right) \exp \left(\left\langle x, \psi\left(t_{n}, u\right)-u\right\rangle\right)-1}{t_{n}} \\
& =\lim _{n \rightarrow \infty} \frac{f_{-u}(x) \mathbb{E}^{x}\left[f_{u}\left(X_{t_{n}}\right)\right]-1}{t_{n}} \\
& =\lim _{n \rightarrow \infty} \frac{1}{t_{n}}\left\{\int_{D} e^{\langle\xi-x, u\rangle} p_{t_{n}}(x, d \xi)-1\right\} \\
& =\lim _{n \rightarrow \infty} \frac{1}{t_{n}}\left\{\int_{D-x}\left(e^{\langle\xi, u\rangle}-1\right) \widetilde{p}_{t_{n}}(x, d \xi)+\frac{p_{t_{n}}(x, D)-1}{t_{n}}\right\},
\end{aligned}
$$

where $p_{t}(x, d \xi)$ is the transition kernel of the Markov process $\left(X_{t}\right)_{t \geq 0}$, and $\widetilde{p}_{t}(x, d \xi)$ is its 'shifted transition kernel' $\widetilde{p}_{t}(x, d \xi):=p_{t}(x, d \xi+x)$. The right hand side of (4.8) can be regarded as a limit of log-characteristic functions of (infinitely divisible) sub-stochastic measures. ${ }^{2}$ That is, there exist infinitely divisible sub-stochastic

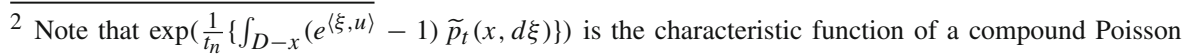
distribution with intensity $\frac{1}{t_{n}}$ and jump measure $\widetilde{p}_{t}(x, d \xi)$.
} 
measures $\mu_{n}(x, d \xi)$, such that

$$
\exp (F(u)+\langle x, R(u)\rangle)=\lim _{n \rightarrow \infty} \int_{\mathbb{R}^{d}} e^{\langle u, \xi\rangle} \mu_{n}(x, d \xi), \quad \text { for all } u \in \mathcal{U}^{\circ}
$$

Let now $\theta \in \mathbb{R}^{d}$ with $\theta_{I}<0$ and $\theta_{J}=0$ (note that $\theta \in \mathcal{U}^{\circ}$ ) and consider the exponentially tilted measures $e^{\langle\theta, \xi\rangle} \mu_{n}(x, d \xi)$. Their characteristic functions converge to $\exp (F(u+\theta)+\langle x, R(u+\theta)\rangle)$. Thus, by Lévy's continuity theorem, there exists $\mu_{*}(x, d \xi)$ such that $e^{\langle\theta, \xi\rangle} \mu_{n}(x, d \xi) \rightarrow \mu_{*}(x, d \xi)$ weakly. On the other hand, by Helly's selection theorem, $\mu_{n}(x, d \xi)$ has a vaguely convergent subsequence, which converges to some measure $\mu(x, d \xi)$. By uniqueness of the weak limit we conclude that $\mu(x, d \xi)=e^{\langle-\theta, \xi\rangle} \mu_{*}(x, d \xi)$. Thus we have that for all $x \in D$ and $u \in \mathcal{U}^{\circ}$ with $\operatorname{Re} u$ in a neighborhood of $\theta$,

$$
\begin{aligned}
\exp (F(u)+\langle x, R(u)\rangle) & =\lim _{n \rightarrow \infty} \int_{\mathbb{R}^{d}} e^{\langle u, \xi\rangle} \mu_{n}(d, d \xi) \\
& =\lim _{n \rightarrow \infty} \int_{\mathbb{R}^{d}} e^{\langle u-\theta, \xi\rangle} e^{\langle\theta, \xi\rangle} \mu_{n}(x, d \xi) \\
& =\int_{\mathbb{R}^{d}} e^{\langle u-\theta, \xi\rangle} \mu_{*}(x, d \xi)=\int_{\mathbb{R}^{d}} e^{\langle u, \xi\rangle} \mu(x, d \xi) .
\end{aligned}
$$

But the choice of $\theta$ was arbitrary, such that (4.9) extends to all $u \in \mathcal{U}^{\circ}$. Applying dominated convergence to the last term of (4.9) shows that both $F$ and $R$ have a continuous extension to all of $\mathcal{U}$, which we also denote by $F$ and $R$ respectively.

It remains to show that (4.7) remains valid on $\mathcal{U}$ : Let $u \in \mathcal{U}$ and $\left(u_{n}\right)_{n \in \mathbb{N}} \in \mathcal{U}^{\circ}$ such that $u_{n} \rightarrow u$. Remember that by Proposition $3.4 u_{n} \in \mathcal{U}^{\circ}$ implies that also $\psi\left(t, u_{n}\right) \in \mathcal{U}^{\circ}$ for any $t \geq 0$. Thus we have

$$
\begin{aligned}
\int_{0}^{t} R(\psi(s, u)) d s & =\int_{0}^{t} \lim _{u_{n} \rightarrow u} R\left(\psi\left(s, u_{n}\right)\right) d s=\lim _{u_{n} \rightarrow u} \int_{0}^{t} R\left(\psi\left(s, u_{n}\right)\right) d s \\
& =\lim _{u_{n} \rightarrow u} \int_{0}^{t} \frac{\partial}{\partial t} \psi\left(s, u_{n}\right) d s=\lim _{u_{n} \rightarrow u} \psi\left(t, u_{n}\right)-u_{n}=\psi(t, u)-u .
\end{aligned}
$$

Since the left hand side of (4.10) is $t$-differentiable, also the right hand side is, and we obtain $R(u)=\left.\frac{\partial}{\partial t} \psi(t, u)\right|_{t=0}$ for all $u \in \mathcal{U}$. A similar calculation as above can be made upon replacing $R$ with $F$, resulting in $F(u)=\left.\frac{\partial}{\partial t} \Phi(t, u)\right|_{t=0}$ for all $u \in \mathcal{U}$, and thus showing that the partially additive affine process $\left(X_{t}\right)_{t \geq 0}$ is regular. 


\section{All affine processes are regular}

In this final section we reduce the question of regularity of general stochastically continuous affine processes to stochastically continuous, partially additive affine processes. Recall that for those processes we have shown regularity in the preceding section. The transformation of general affine processes to partially additive processes is based on the method of the moving frame, which has been successfully applied in the context of SPDEs; see for instance [5] and [6].

Theorem 5.1 Every stochastically continuous affine process $X$ is regular.

Proof By Theorem 3.5 X is a Feller process, and thus has a càdlàg version. Clearly, choosing a càdlàg version will not alter the functions $\Phi(t, u)$ and $\psi(t, u)$ defined by (2.1). Furthermore, we know by Proposition 3.3 that

$$
\psi_{J}(t, u)=\exp (t \beta) u_{J}
$$

for $(t, u) \in \mathcal{Q}$ and a real $n \times n$ matrix $\beta$. We define the $d \times d$ matrix

$$
K=\left(\begin{array}{cc}
\operatorname{id}_{m} & 0 \\
0 & \beta
\end{array}\right)
$$

and the transformation $\mathcal{T}$

$$
Z_{t}=\mathcal{T}[X]_{t}:=X_{t}-K^{\top} \int_{0}^{t} X_{s} d s
$$

transforming the process $X$ path-by-path into a process $Z$. Note that the transformation is well-defined due to the càdlàg property of the trajectories, and preserves the stochastic continuity of $X$. Moreover, the transformation can be inverted by

$$
\mathcal{T}^{-1}[Z]_{t}=Z_{t}+K^{\top} \int_{0}^{t} \exp \left((t-s) K^{\top}\right) Z_{s} d s
$$

which is seen directly by inserting (5.3) and integrating by parts.

We claim that the transformed process $Z=\mathcal{T}[X]$ is a partially additive affine process. For this purpose we calculate the conditional characteristic function: Let $u \in i \mathbb{R}^{d}$, and for each $N \in \mathbb{N}$ and $k \in\{0, \ldots, N\}$, define $t_{k}=k t / N$, such that $t_{0}, \ldots, t_{N}$, is an equidistant partition of $[0, t]$ into intervals of mesh $t / N$. Writing the time-integral as 
a limit of Riemann sums, and using dominated convergence we obtain

$$
\begin{aligned}
& \mathbb{E}^{x}\left[\exp \left(\left\langle u, Z_{t+s}\right\rangle\right) \mid \mathcal{F}_{s}\right]=\exp \left(-\left\langle u, K^{\top} \int_{0}^{s} X_{r} d r\right\rangle\right) \\
& \cdot \lim _{N \rightarrow \infty} \mathbb{E}^{x}\left[\exp \left(\left\langle u, X_{t+s}\right\rangle-\frac{t}{N}\left\langle K u, \sum_{k=0}^{N-1} X_{s+t_{k}}\right\rangle\right) \mid \mathcal{F}_{s}\right] .
\end{aligned}
$$

With the shorthands $h:=t / N$ and $\Sigma_{n}:=\sum_{k=0}^{n} X_{s+t_{k}}$, and using the tower law as well as the affine property of $\left(X_{t}\right)_{t \geq 0}$, the expectation on the right side can be written as

$$
\begin{aligned}
\mathbb{E}^{x} & {\left[\exp \left(\left\langle u, X_{t+s}\right\rangle-h\left\langle K u, \Sigma_{N-1}\right\rangle\right) \mid \mathcal{F}_{s}\right] } \\
= & \mathbb{E}^{x}\left[\exp \left(-h\left\langle K u, \Sigma_{N-2}\right\rangle\right) \cdot \mathbb{E}^{x}\left[\exp \left(\left\langle\left(\operatorname{id}_{d}-h K\right) u, X_{t+s}\right\rangle\right) \mid \mathcal{F}_{s+t_{N-1}}\right] \mid \mathcal{F}_{s}\right] \\
= & \Phi\left(h,\left(\operatorname{id}_{d}-h K\right) u\right) \\
& \cdot \mathbb{E}^{x}\left[\exp \left(\left\langle\psi\left(h,\left(\operatorname{id}_{d}-h K\right) u\right), X_{s+t_{N-1}}\right\rangle-h\left\langle K u, \Sigma_{N-2}\right\rangle\right) \mid \mathcal{F}_{s}\right] .
\end{aligned}
$$

Applying the tower law $(N-1)$-times in the same way (and conditioning on $\mathcal{F}_{S+t_{N-1}}, \mathcal{F}_{S+t_{N-2}}, \ldots, \mathcal{F}_{S+t_{1}}$, respectively) we arrive at the equation

$$
\begin{aligned}
& \mathbb{E}^{x}\left[\exp \left(\left\langle u, X_{t+s}\right\rangle-\frac{t}{N}\left\langle K u, \sum_{k=0}^{N-1} X_{s+t_{k}}\right\rangle\right) \mid \mathcal{F}_{s}\right] \\
& =p(N-1 ; t, u) \exp \left(\left\langle X_{s}, q(N-1 ; t, u)\right\rangle\right),
\end{aligned}
$$

where the quantities $p(N-1 ; t, u)$ and $q(N-1 ; t, u)$ are defined through the following recursion:

$$
\begin{aligned}
& p(0 ; t, u)=1, \quad p(k+1 ; t, u)=\Phi\left(h,\left(\operatorname{id}_{d}-h K\right) q(k ; t, u)\right) \cdot p(k ; t, u), \\
& q(0 ; t, u)=u, \quad q(k+1 ; t, u)=\psi\left(h,\left(\operatorname{id}_{d}-h K\right) q(k ; t, u)\right),
\end{aligned}
$$

Since the Riemann sums in (5.5) converge point by point, we conclude that the quantities $p(N-1 ; t, u)$ and $q(N-1 ; t, u)$ converge to some functions $p(t, u), q(t, u)$ as $N \rightarrow \infty$, and thus that

$$
\mathbb{E}^{x}\left[\exp \left(\left\langle u, Z_{t+s}\right\rangle\right) \mid \mathcal{F}_{s}\right]=p(t, u) \exp \left(\left\langle q(t, u), X_{s}\right\rangle-\left\langle u, K^{\top} \int_{0}^{s} X_{r} d r\right\rangle\right)
$$


for all $t, s \geq 0$ and $u \in i \mathbb{R}^{d}$. Let now $q_{J}(t, u)$ denote the $J$-components of $q(t, u)$. Based on the recursion (5.6) and the fact that $\psi_{J}(t, u)=e^{\beta t} u_{J}$ it holds that

$$
q_{J}(t, u)=\lim _{N \rightarrow \infty} e^{t \beta}\left(\operatorname{id}_{n}-\frac{t \beta}{N}\right)^{N-1} u_{J}=e^{t \beta} e^{-t \beta} u_{J}=u_{J}
$$

Thus we can rewrite (5.7) as

$$
\mathbb{E}^{x}\left[\exp \left(\left\langle u, Z_{t+s}\right\rangle\right) \mid \mathcal{F}_{s}\right]=p(t, u) \exp \left(\left\langle q_{I}(t, u), Z_{s}^{I}\right\rangle+\left\langle u_{J}, Z_{s}^{J}\right\rangle\right)
$$

which shows that $Z$ is indeed a stochastically continuous, partially additive affine process. By Theorem 4.3 such a process is regular. Hence, the functions $\widetilde{F}(u)=$ $\left.\frac{\partial}{\partial t} p(t, u)\right|_{t=0}$ and $\widetilde{R}(u)=\left.\frac{\partial}{\partial t} q(t, u)\right|_{t=0}$ exist and satisfy the admissibility conditions in [3, Def. 2.6]. By [3, Thm. 2.7], the functions $\widetilde{F}(u)$ and $\widetilde{R}(u)+K u$ are also admissible, and thus define a regular affine process $\widetilde{X}$. Using now the Feynman-Kac formula in [3, Prop. 11.2], it is seen that the transformation $\mathcal{T}$ transforms the regular affine process $\widetilde{X}$ into the regular affine process $\mathcal{T}[\widetilde{X}]$ characterized by $\widetilde{F}(u)$ and $\widetilde{R}(u)$-that is into a process equal in law to $Z$. We have shown that

$$
\mathcal{T}[X]=Z \text { and } \mathcal{T}[\tilde{X}]=Z,
$$

where equality is understood in law. Since the transformation $\mathcal{T}$ can be inverted pathby-path, we conclude that $X=\widetilde{X}$ in law, and thus that $X$ is regular.

Remark 5.2 The intuition behind the 'moving frame' transformation is the following: given the process $X$ we first construct a time-dependent transformation

$$
Y_{t}=\exp \left(-K^{\top} t\right) X_{t}
$$

the 'moving frame'. In the moving frame the process $X$ becomes time-inhomogeneous, but can be re-scaled (in order to arrive at a time-homogeneous process) by the stochastic integral

$$
d Z_{t}=\exp \left(K^{\top} t\right) d Y_{t}
$$

The stochastic integral can be defined by integration by parts, i.e.,

$$
Z_{t}=\exp \left(K^{\top} t\right) Y_{t}-K^{\top} \int_{0}^{t} \exp \left(K^{\top} t\right) Y_{r} d r
$$

which yields the transformation formula (5.3). The method of the moving frame is therefore an operation which allows to remove (or change) the linear drift of an affine process. 
Remark 5.3 Now that we have shown that every stochastically continuous affine process $X$ is regular, all the results of [3] on regular affine processes apply to $X$. It follows for example that the set $\mathcal{Q}$, introduced in (2.4) is actually equal to $\mathcal{U}$, and in particular simply connected. Thus, the $\log a r i t h m ~ \phi(t, u)=\log \Phi(t, u)$ is uniquely defined by choosing the main branch of the complex logarithm, and we can write

$$
\mathbb{E}^{x}\left[e^{\left\langle X_{t}, u\right\rangle}\right]=\exp (\phi(t, u)+\langle x, \psi(t, u)\rangle)
$$

for all $(t, u) \in \mathcal{U}$, as in [3].

\section{References}

1. Aczél, J.: Functional Equations and their Applications. Academic Press, New York (1966)

2. Dawson, D.A., Li, Z.: Skew convolution semigroups and affine Markov processes. Ann. Probab. 34(3), 1103-1142 (2006)

3. Duffie, D., Filipovic, D., Schachermayer, W.: Affine processes and applications in finance. Ann. Appl. Probab. 13(3), 984-1053 (2003)

4. Filipović, D., Teichmann, J.: Regularity of finite-dimensional realizations for evolution equations. J. Funct. Anal. 197, 433-446 (2003)

5. Filipović, D., Tappe, S., Teichmann, J.: Jump-diffusions in Hilbert spaces: existence, stability and numerics, Stochastics (2010, forthcoming). arXiv/0810.5023

6. Filipović, D., Tappe, S., Teichmann, J.: Term structure models driven by Wiener process and Poisson measures: existence and positivity, SIAM J. Financ. Math (2010, forthcoming). arXiv/0905.1413

7. Jacob, N.: Pseudo Differential Operators and Markov Processes, vol. I. Imperial College Press, London (2001)

8. Kawazu, K., Watanabe, S.: Branching processes with immigration and related limit theorems. Theory Probab. Appl. XVI(1), 36-54 (1971)

9. Keller-Ressel, M.: Moment explosions and long-term behavior of affine stochastic volatility models. Math. Finance (2008, forthcoming). arXiv:0802.1823

10. Keller-Ressel, M.: Affine processes-contributions to theory and applications. PhD thesis, TU Wien (2008)

11. Lukacs, E.: Characteristic Functions. Charles Griffin \& Co Ltd., London (1960)

12. Montgomery, D., Zippin, L.: Topological Transformation Groups. Interscience, New York (1955)

13. Rogers, L.C.G., Williams, D.: Diffusions, Markov Processes and Martingales, 2nd edn, vol. 1. Cambridge Mathematical Library, Cambridge (1994)

14. Semadeni, Z.: Banach Spaces of Continuous Functions. Polish Scientific Publishers, Poland (1971) 